\title{
USO DE FRAGMENTOS DE CARAPACA PARA MONITORAMENTO DO MERCÚRIO EM DUAS ESPÉCIES DE TARTARUGAS MARINHAS NO NORDESTE DO BRASIL
}

\author{
Use of carapace for monitoring mercury in two species \\ of sea turtles in northeast of Brazil
}

\author{
César Augusto Barrios Rodriguez ${ }^{1 *}$, Moises Fernandes Bezerra², Luiz Drude de Lacerda ${ }^{3}$ \\ ${ }^{1}$ Doutorando do Instituto de Ciências do Mar, Universidade Federal do Ceará, Bolsista da Fundação Cearense de Apoio \\ ao Desenvolvimento Científico e Tecnológico (FUNCAP), Av. da Abolição 3207, Fortaleza, CE 60165-081. \\ E-mail: hpbarrios15@gmail.com \\ ${ }^{2}$ Doutorando da Universidade de San Diego, Califórnia, USA. E-mail: mmoisesfb@hotmail.com \\ ${ }^{3}$ Professor do Instituto de Ciências do Mar, Universidade Federal do Ceará, Av. da Abolição 3207, Fortaleza, \\ CE 60165-081. E-mail: Idrude@pq.cnpq.br \\ *autor para correspondência
}

\begin{abstract}
RESUMO
Este trabalho compara concentrações de $\mathrm{Hg}$ para duas espécies de tartarugas marinhas, Caretta caretta e Chelonia mydas, encontradas na zona costeira do nordeste brasileiro e fazendo uso de fragmentos de carapaça como método não invasivo de monitoramento dos níveis ambientais de $\mathrm{Hg}$. Os resultados não mostraram diferença significativa entre as concentrações de $\mathrm{Hg}$ nas duas espécies. Porém, a comparação entre a $C$. caretta (adultos) e C. mydas (juvenis e subadulto), permitiu observar que a dieta exerce influência na concentração de $\mathrm{Hg}$ nesses organismos. Tornando relevante, em futuros estudos, a quantificação de $\mathrm{Hg}$ tanto nas espécies em estudo quanto em sua fonte alimentar.
\end{abstract}

Palavras-chave: Caretta caretta; Chelonia mydas; mercúrio; biomonitoramento; carapaça.

\begin{abstract}
This work compares the concentrations of $\mathrm{Hg}$ in two species of sea turtles, Caretta caretta and Chelonia mydas, found in the coastal zone of northeastern Brazil using carapace fragments as
\end{abstract}

Recebido em: 04/07/2018

Aprovado em: 23/07/2018

Publicado online em: 05/09/2018 
a non-invasive method for monitoring environmental levels of $\mathrm{Hg}$. The results show no significant difference between the $\mathrm{Hg}$ concentrations in the two species. However, the comparison between $\mathrm{C}$. caretta (adults) and C. mydas (juveniles and subadult) allowed us to observe that diet exerts influence on the concentration of $\mathrm{Hg}$ in these organisms, making relevant in future studies, the quantification of $\mathrm{Hg}$ in both, the species and its food source.

Key words: Caretta caretta; Chelonia mydas; mercury; biomonitoring; carapace.

\section{INTRODUÇÃO}

Grandes quantidades de $\mathrm{Hg}$ estão sendo acumulados no ambiente, e o oceano, devido a sua superfície, é o receptor de aproximadamente $90 \%$ do Hg incorporado através da deposição húmida e seca da atmosfera (Mason et al., 1994). Atualmente as emissões antrópicas e naturais de $\mathrm{Hg}$ continuam contaminando oceanos, bacias hidrográficas e sedimentos de rios e lagos (UNEP, 2013). O Hg depositado na forma inorgânica, essencial para a produção da sua forma mais tóxica, o metil mercúrio $\left(\mathrm{CH}_{3} \mathrm{Hg}^{+}\right)$, durante décadas ou séculos pode ficar disponível em compartimentos ambientais acessíveis a biota local, antes dele ser removido por processos naturais para depósitos permanentes, geralmente sedimentos (Hong et al., 2012; Schneider et al., 2013). O longo tempo residência do $\mathrm{Hg}$ nos oceanos (30 anos), entretanto, e sua alta toxicidade, tornam particularmente importante o estudo de sua distribuição em espécies marinhas de longo tempo de vida (UNEP, 2013).

Existe a necessidade de identificar espécies adequadas que exibam ampla distribuição geográfica e que possam ser utilizadas como biomonitores. O uso de tais organismos, permitiria que as comparações absolutas das concentrações de $\mathrm{Hg}$ acumuladas sejam feitas em grandes áreas geográficas (Rainbow \& Phillips, 1993). Nesse contexto, as tartarugas marinhas apresentam características essenciais para serem consideradas biomonitores cosmopolitas. Seu longo tempo de vida, sua diversificada dieta alimentar, distribuição circunglobal e a capacidade de apresentar altas concentrações de Hg sem ter sua saúde afetada de forma imediata, permitem avaliar as concentrações de $\mathrm{Hg}$ em grandes áreas oceânicas.

O Brasil, com um litoral de aproximadamente $8000 \mathrm{~km}$ de extensão, apresenta zonas importantes para a alimentação e desova de cinco das sete espécies de tartarugas marinhas conhecidas no mundo (Menezes, 1972). Dentre as cinco espécies encontradas no Brasil, Caretta caretta (Linnaeus, 1758) (tartaruga cabeçuda) e Chelonia mydas (Linnaeus, 1758) (tartaruga verde) apresentam uma das maiores populações conhecidas para o Atlântico Sudoeste. Além disso, estudos de genética mostram que a população brasileira de tartaruga cabeçuda e tartaruga verde apresentam diferenças das demais populações conhecidas no mundo (Santana et al., 2011). As características mencionadas, permitiriam o uso de tais organismos para monitorar a presença de metais como o $\mathrm{Hg}$ nas zonas costeiras da Bahia e do Ceará, por serem áreas importantes na desova e alimentação destas tartarugas respectivamente.

$\mathrm{O}$ uso de tartarugas marinhas como biomonitores das concentrações de $\mathrm{Hg}$ no Atlântico Sudoeste, implicaria o uso de métodos não invasivos que permitam a realização deste tipo de trabalho, em espécies ameaçadas e, portanto, protegidas por lei. Um destes métodos é o uso de estruturas queratinizadas. O alto teor de aminoácidos em estruturas como a carapaça em quelônios, facilita a existência de sítios potencias para a ligação do Hg. Como resultado, este metal pode ficar imobilizado nas camadas da queratina e assim re- 
fletir a exposição ao longo do tempo (Toni et al., 2007). Vários estudos vêm avaliando o uso da carapaça das tartarugas marinhas como um possível método de monitoramento ambiental do $\mathrm{Hg}$, sendo as tartarugas verde e cabeçuda as mais utilizadas para este tipo de trabalho. Nesse sentido o principal objetivo deste trabalho é fazer uma comparação entre as concentrações de Hg para duas espécies de tartarugas marinhas (verde e cabeçuda), encontradas na zona costeira do nordeste brasileiro e fazendo uso de fragmentos de carapaça como método não invasivo de monitoramento.

\section{MATERIAL E MÉTODOS}

Todos os procedimentos e análises deste trabalho foram realizados dentro das normas vigentes da legislação ambiental brasileira, efetuados sob autorização do Sistema de Autorização e Informação em Biodiversidade - SISBIO, Licença No 21693-9 (2016).

As coletas dos fragmentos de carapaça foram realizadas no Ceará (Praia Almofala e Volta do Rio) e Bahia (Praia do Forte e Camaçari) (Fig. 1), durante os anos 2009, 2010 e 2016. Foram amostrados 32 indivíduos, sendo 16 da espécie C. caretta (8 no Ceará e 8 na Bahia) e 16 de C. mydas (8 no Ceará e 8 na Bahia). Todos os indivíduos foram classificados como juvenis, adultos e sub adultos de acordo com Dodd (1988) e Almeida et al. (2011). Os fragmentos foram coletados de forma aleatória em diferentes regiões da carapaça. Também foram mensuradas o tamanho dos indivíduos, comprimento curvilíneo da carapaça (CCC) e a largura curvilínea da carapaça (LCC).

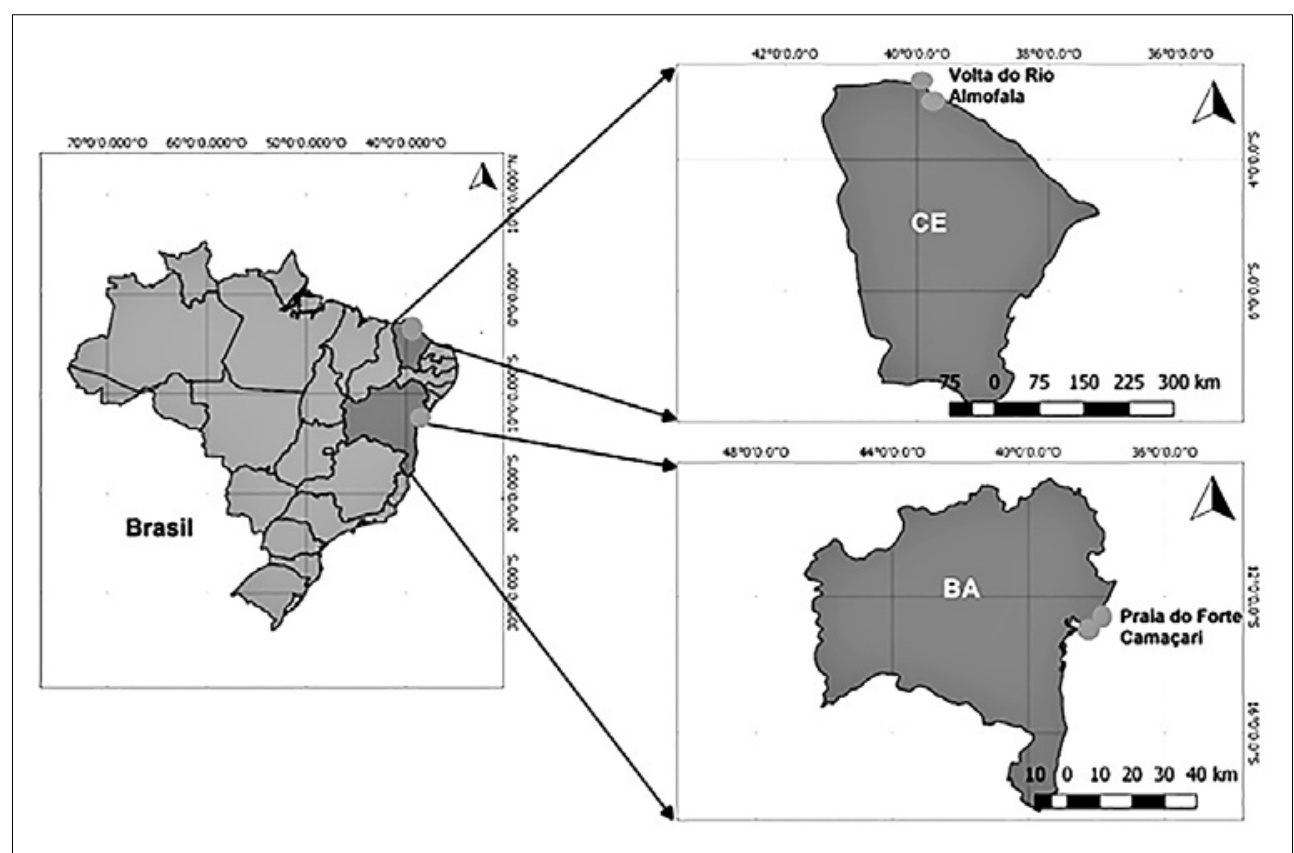

Figura 1 - Área de estudo no litoral do estado da Bahia (Praia do Forte e Camaçari) e no litoral do Ceará (Praia Almofala e Volta do Rio).

A metodologia utilizada para a quantificação do $\mathrm{Hg}$ total nas amostras biológicas seguiu aquela adaptada de Bezerra et al. (2012), que consistiu na pesagem das amostras em duplicata, com peso de $0,5 \mathrm{~g}$, em tubos de teflon, sendo, posteriormente, adicionados $10 \mathrm{~mL}$ de ácido nítrico concentrado $\left(\mathrm{HNO}_{3} 65 \%\right)$ em uma pré-digestão de 1 h. Após a pré- 
digestão, a digestão foi realizada em forno digestor micro-ondas com potência de $800 \mathrm{~W}$ e temperatura de $200{ }^{\circ} \mathrm{C}$ durante $30 \mathrm{~min}$. Em seguida, $1 \mathrm{~mL}$ de peróxido de hidrogênio $\left(\mathrm{H}_{2} \mathrm{O}_{2}\right)$ foi adicionado. $\mathrm{O}$ extrato final foi transferido para balões volumétricos de $100 \mathrm{~mL}$, aferindo-os com água destilada. A quantificação do $\mathrm{Hg}$ nas amostras foi executada em um espectrofotômetro de absorção atômica por geração de vapor frio (CV-ASS), modelo NIC RA-3 da NIPPON.

O teste de Shapiro Wilk foi utilizado para avaliar a normalidade dos dados. O teste não paramétrico Mann-Whitney foi aplicado para avaliar as diferenças nas concentrações de $\mathrm{Hg}$ total entre as espécies C. caretta e C. mydas do Ceará e da Bahia. O teste $\mathrm{t}$ de Student foi utilizado para avaliar as diferenças no comprimento curvilíneo da carapaça. Quando encontrados valores outliers, eles foram retirados para uma melhor interpretação dos dados. O valor de significância utilizado para os testes foi de $95 \%(p<0,05)$. Os testes estatísticos e a elaboração dos gráficos foram realizados utilizando os softwares RStudio (versão 0.98.976 - () RStudio, Inc. 2009-2013) e Microsoft Office 365.

\section{RESULTADOS}

A distribuição dos tamanhos dos indivíduos analisados sugere que para C. caretta foram amostrados indivíduos adultos enquanto que para C. mydas foram amostrados indivíduos juvenis.

As amostras de $C$. caretta do Ceará mostraram uma variação no tamanho na faixa de 78,7 a $107 \mathrm{~cm}$ de CCC (95 $\pm 10 \mathrm{~cm})$, enquanto que os indivíduos da Bahia apresentaram variações de 87,1 a 106,9 cm de CCC $(97,5 \pm 6 \mathrm{~cm}$ ) (Figura 2, c). A comparação entre os tamanhos das tartarugas cabeçudas da Bahia e do Ceará não mostraram diferença significativa $(\mathrm{t}=0,56 ; \mathrm{p}>0,05)$. A espécie $C$. mydas na região costeira do Ceará mostrou um CCC variando de $38 \mathrm{~cm}$ a 55,3 cm $(44,3 \pm 6 \mathrm{~cm})$, enquanto que os indivíduos da Bahia mostraram um comprimento variando de 36,2 a $62 \mathrm{~cm}$ de CCC $(45,1 \pm 9 \mathrm{~cm})$ (Figura 2, a). A comparação dos tamanhos das tartarugas verdes para essas duas áreas não mostrou diferença significativa $(\mathrm{t}=-0,05 ; \mathrm{p}>0,05)$. Uma comparação entre o CCC de $C$. mydas e $C$. caretta do Ceará e da Bahia, entretanto mostrou diferença significativa $(t=13,8 ; p<0.05)$, sendo a $C$. caretta do Ceará e da Bahia significativamente maiores que mostraram os maiores que $C$. mydas (Figura 3, a e c).

As concentrações de $\mathrm{Hg}$ total em C. caretta do Ceará variaram de 33,5 a $402,5{\mathrm{ng} . \mathrm{g}^{-1}}^{-1}$ $\left(207,7 \pm 169\right.$ ng. $\left.{ }^{-1}\right)$, e nos indivíduos da Bahia de 12 a 291 ng. $\mathrm{g}^{-1}\left(86,7 \pm 95\right.$ ng. $\left.\mathrm{g}^{-1}\right)$ (Figura 2, d). As concentrações de $\mathrm{Hg}$ total das C. caretta do Ceará e da Bahia não apresentaram diferença significativa $(\mathrm{U}=16 ; \mathrm{p}>0,05)$.

C. mydas no Ceará apresentou concentrações de $\mathrm{Hg}$ variando de 8,9 a 447,3 ng.g-1 $\left(159,3 \pm 183\right.$ ng. $\left.\mathrm{g}^{-1}\right)$, enquanto que na Bahia, os indivíduos mostraram concentrações de $\mathrm{Hg}$ variando de144,3 a 199 ng.g $^{-1}\left(19,3 \pm 471,7\right.$ ng.g ${ }^{-1}$ ) (Figura 2, b). Entre concentrações de Hg total nas tartarugas cabeçudas e verdes da Bahia, não ocorreram diferença significativa (U $=27 ; \mathrm{p}>0,05)$. Também não foram encontradas diferenças significativas entre $C$. mydas e $C$. caretta na Bahia $(U=37 ; p>0,05)$ e no Ceará $(U=48 ; p>0,05)$ (Figura 3, b e d). 


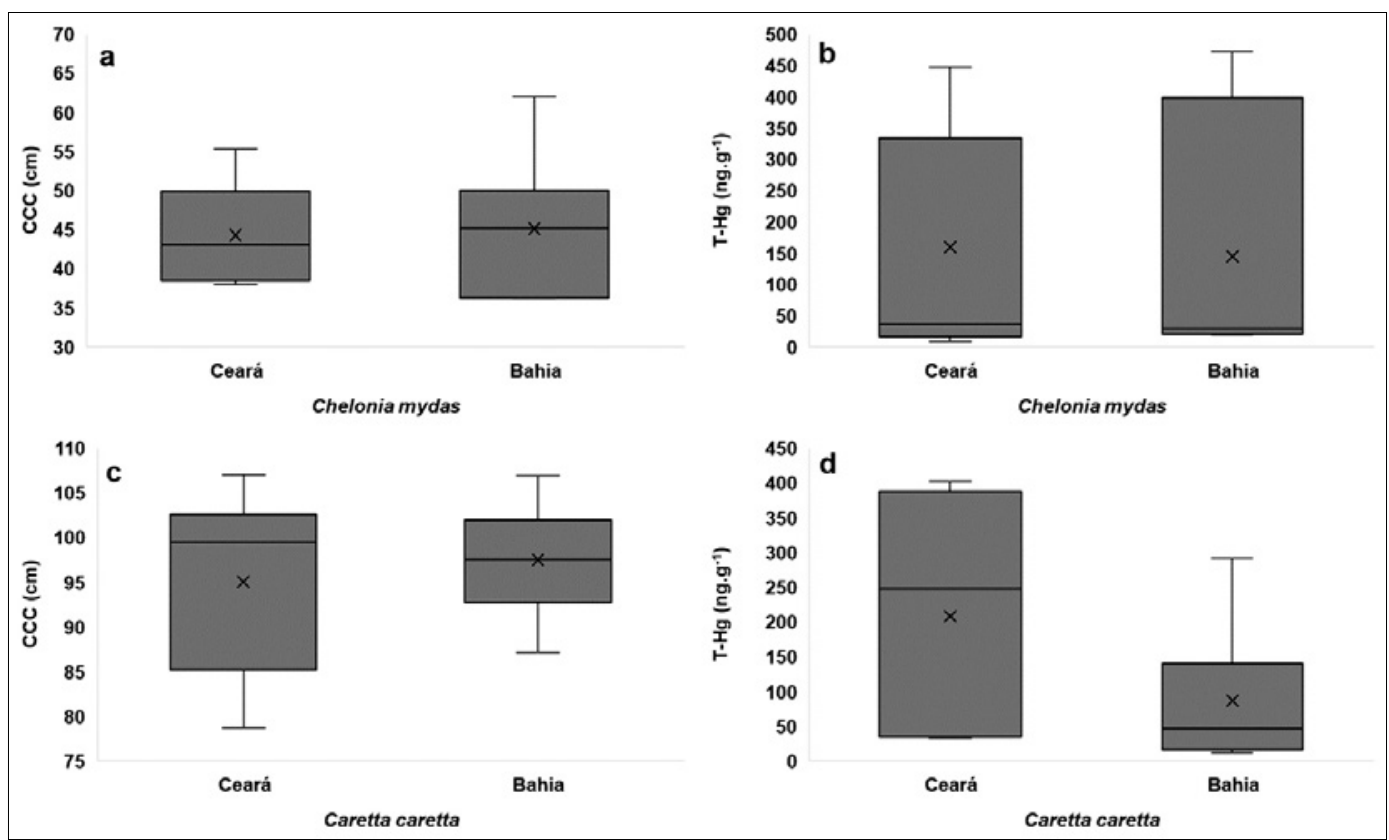

Figura 2-Comprimento curvilíneo da carapaça (CCC) (a) e concentração de Hg total para C. mydas (b); Comprimento curvilíneo da carapaça (CCC) (c) e concentração de Hg total em C. caretta (d).

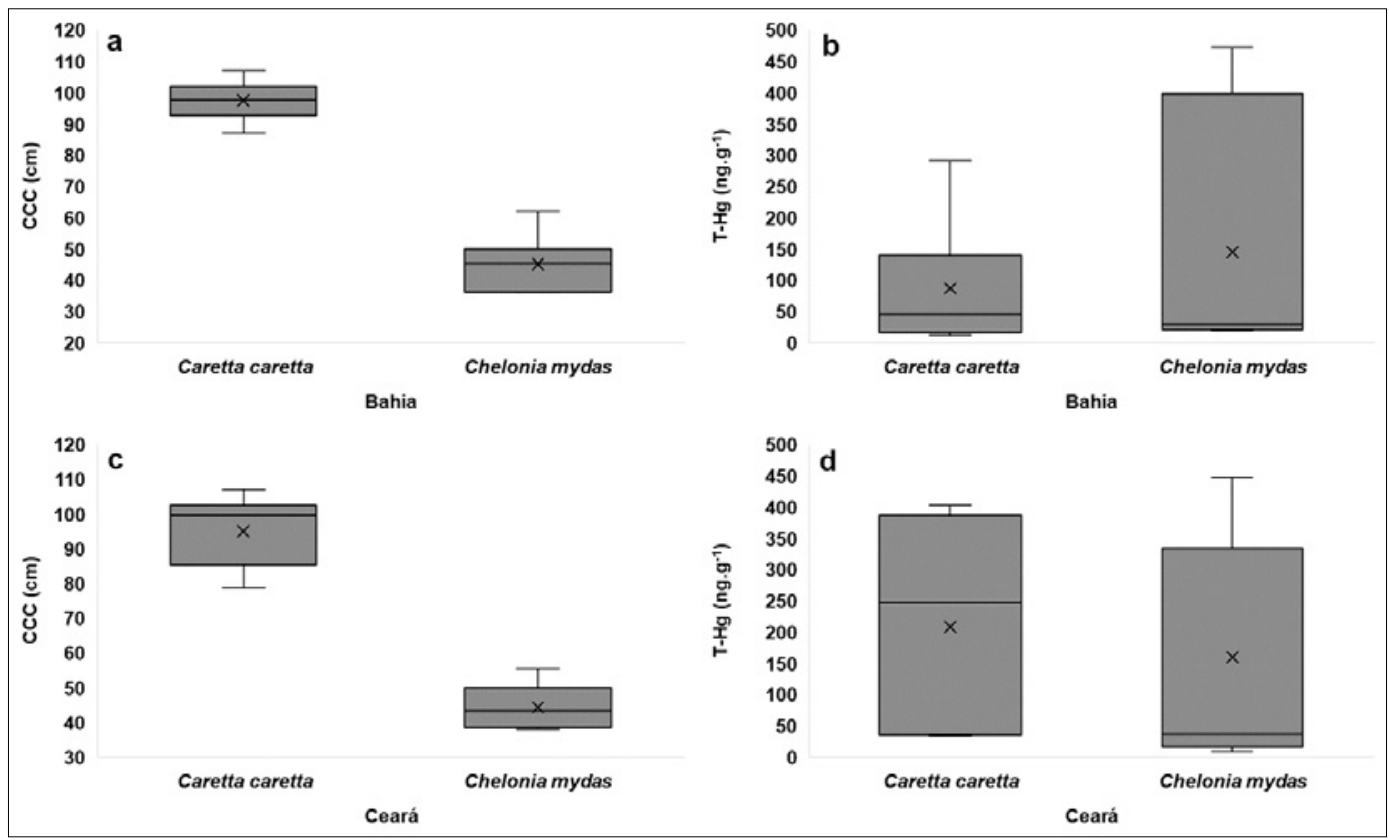

Figura 3 - Comprimento curvilíneo da carapaça (CCC) (a) e concentração de Hg total para C. caretta e C. mydas do litoral baiano (b). Comprimento curvilíneo da carapaça (CCC) (c) e concentração de Hg total para C. caretta e C. mydas do litoral cearense (d).

\section{DISCUSSÃO}

Na atualidade é conhecida a existência de duas subpopulações de tartarugas cabeçudas e verdes no Brasil, e que apresentam áreas de alimentação e reprodução diferentes. No caso das tartarugas utilizadas neste trabalho as mesmas pertencem à subpopulação do Nordeste, onde as áreas de alimentação estão no litoral cearense e as respectivas 
áreas de reprodução estão no litoral baiano (Marcovaldi, 2010). Estas duas áreas exibem diferentes níveis de intervenção antrópica o que repercute na entrada de diferentes tipos de metais tóxicos, e também nos metais que podem ser absorvidos pelas tartarugas (Bezerra et al., 2015). Embora o litoral baiano apresente maior desenvolvimento industrial próximo à costa e por consequência maiores concentrações de Hg nas águas costeiras (Marins et al., 2004; Rocha et al., 2012), esta diferença não foi encontrada comparando as C. caretta e C. mydas amostradas em ambos litorais.

Diferente deste trabalho, Bezerra et al. (2015) encontrou maiores concentrações de Hg em tartarugas verdes da Bahia comparado com as tartarugas verdes do litoral cearense. Esta diferença foi explicada pelas maiores concentrações de $\mathrm{Hg}$ em presas que compõem a dieta dos organismos coletados no litoral da Bahia em relação aos indivíduos coletados no Ceará (Bezerra et al., 2015). A preferência alimentar, frequência do consumo e o conteúdo de $\mathrm{Hg}$ que esses itens possam apresentar, são pontos de extrema importância a ser avaliados. Bezerra et al. (2015), também observou que os indivíduos podem mostrar preferências alimentares semelhantes, porém, variáveis concentrações de $\mathrm{Hg}$ em diferentes tipos de tecidos, refletindo uma possível influência do $\mathrm{Hg}$ encontrado no meio.

É importante ressaltar que das sete espécies de tartarugas marinhas, seis são altamente migratórios, como é o caso das espécies utilizadas neste trabalho. Intuitivamente, essa mobilidade resulta em exposição a diferentes regimes de contaminantes, tornando difíceis as relações entre a contaminação do $\mathrm{Hg}$ com uma área determinada (Day et al., 2005), além disso existem fatores biológicos, ecológicos e inclusive as próprias características da carapaça que influenciam na forma em que essa estrutura pode incorporar o $\mathrm{Hg}$. A capacidade da carapaça para refletir as concentrações de $\mathrm{Hg}$ pode variar de uma espécie para outra, devido à existência de diferenças na composição da queratina e ao crescimento diferencial das placas (Day et al., 2005; Toni et al., 2007). A espessura dessas placas varia dependendo da sua localização na carapaça (vertebral, lateral e marginais). Dessa forma, os fragmentos de carapaça que são coletados em diferentes profundidades e localizações podem refletir diferentes períodos de deposição de $\mathrm{Hg}$ na vida da tartaruga (Day et al., 2005).

Uma das principais vias de entrada do $\mathrm{Hg}$ nos organismos é a dieta alimentar, e esta pode variar dependendo de seu estágio de vida (Gray, 2002; Mackay \& Fraser, 2000). Com o uso de diferentes nichos ecológicos no oceano e uma alimentação em diferentes níveis tróficos, a tartaruga cabeçuda e a tartaruga verde são um exemplo dessa variação. Dessa forma a comparação entre esses indivíduos permitiu observar a influência da dieta alimentar nas concentrações do $\mathrm{Hg}$. Os resultados encontrados, mostraram que indivíduos carnívoros podem apresentar concentrações de Hg semelhantes. A C. caretta, espécie conhecida por ser carnívora durante toda sua vida, e a C. mydas, espécie com habito alimentar carnívoro durante a fase juvenil e habito herbívoro durante a fase adulta (Bjorndal, 1997; Sakai et al., 2000; Bezerra et al., 2012), não mostraram diferenças significativas nas concentrações de $\mathrm{Hg}$. Como verificado neste trabalho foram utilizados indivíduos de tartaruga verde na fase juvenil e subadulto, por conseguinte com uma dieta carnívora, pelo que as concentrações de $\mathrm{Hg}$ desses indivíduos, resultaram ser próximos ou semelhantes aos mostrados pela tartaruga cabeçuda. Dessa forma, a variação no conteúdo de Hg que exibem os itens alimentares próprios de uma espécie, irá refletir na concentração de $\mathrm{Hg}$ dessa espécie.

A comparação entre as duas áreas neste estudo foi baseada em um total de 32 indivíduos encontrados em ambas zonas, portanto, seria ideal realizar uma comparação com um número amostral maior, que permitisse ter uma ideia mais clara sobre uma eventual possível diferença nas concentrações de $\mathrm{Hg}$ em C. caretta e C. mydas destas zonas costeiras. E embora não se encontraram diferenças no nível de $\mathrm{Hg}$ que pode apresentar a tartaruga 
cabeçuda e a tartaruga verde, foi possível observar que a dieta, e o estágio de vida, são fatores importantes a serem avaliados em estudos comparativos entre espécies em diferentes regiões.

\section{CONCLUSÃO}

Os resultados apresentados neste trabalho, são um dos primeiros em utilizar duas espécies de tartarugas marinhas para monitorar a presença do Hg em duas zonas costeiras do Brasil, e utilizando fragmentos de carapaça como método não invasivo. Com o número de indivíduos utilizados não foi possível determinar claramente se existe diferenças nas concentrações de $\mathrm{Hg}$ para essas zonas costeiras, porém, foi possível observar que a dieta alimentar é um fator que tem forte influência nas concentrações de $\mathrm{Hg}$ como observado nas tartarugas verdes. Em trabalhos futuros é necessário um número amostral maior que permita ter uma ideia mais clara sobre a presença deste contaminante tanto no litoral cearense como o baiano.

Agradecimento - A FUNCAP pelo suporte financeiro para o desenvolvimento deste trabalho. À equipe do laboratório de biogeoquímica costeira pelo suporte no processo de analises das amostras, e à equipe do Projeto de Conservação de Tartarugas Marinhas (TAMAR), pelo suporte no trabalho de campo.

\section{REFERÊNCIAS BIBLIOGRÁFICAS}

Bezerra, M.F., Lacerda, L.D., Costa, B.G.B. \& Lima, E.H.S.M. Mercury in the sea turtle Chelonia mydas (Linnaeus, 1958) from Ceara coast, NE Brazil. An. Acad. Bras. Cienc. v. 84, p.123-128, 2012.

Bezerra, M.F., Lacerda, L.D., Rezende, C.E., Franco, M.A.L., Almeida, M.G., Macêdo, G.R., Pires, T.T., Rostán, G. \& Lopez, G.G. Food preferences and Hg distribution in Chelonia mydas assessed by stable isotopes. Environ. Pollut., v. 206, p. 236-246, 2015.

Day, R.D., Christopher, S.J., Becker, P.R. \& Whitaker, D.W. Monitoring mercury in the loggerhead sea turtle, Caretta caretta. Environ. Sci. Technol. v.39, p.437-446, 2005.

Dodd, K.C. Synopsis of the biological data on the loggerhead sea turtle Caretta caretta (Linnaeus 1758), US Fish and Wildlife Service, 1988.

de Pádua Almeida, A., Santos, A. J. B., Thomé, J. C. A., Belini, C., Baptistotte, C., Marcovaldi, M. Â. \& Lopez, M.. Avaliação do estado de conservação da tartaruga marinha Chelonia mydas (Linnaeus, 1758) no Brasil. Biodiversidade Brasileira, v.1., 2011.

dos Santos, A. S., Soares, L., Marcovaldi, M. Â., da Silveira Monteiro, D., Giffoni, B. \& de Pádua Almeida, A. Avaliação do estado de conservação da tartaruga marinha Caretta caretta Linnaeus, 1758 no Brasil. Biodiversidade Brasileira, v. 1, 2011.

Gray, J.S. Biomagnification in marine systems: The perspective of an ecologist. Mar. Pollut. Bull. v. 45, p. 46-52, 2002.

Hong, Y. S., Kim, Y. M. \& Lee, K. E. Methylmercury exposure and health effects. Journal of Preventive Medicine and Public Health, v.45, n.6, p.353, 2012. 
Mackay, D. \& Fraser, A. Bioaccumulation of persistent organic chemicals: mechanisms and models., v. 110, p. 375-391, 2000.

Marcovaldi, M.Â., Lopez, G.G., Soares, L.S., Lima, E.H.S.M., Thomé, J.C.A. Almeida, A.P. Satellite-tracking of female loggerhead turtles highlights fidelity behavior in northeastern Brazil. Endanger. Species Res. v.12, p. 263-272, 2010.

Marins, R. V., Paula Filho, F. J. D., Maia, S. R. R., Lacerda, L. D. D. \& Marques, W. S. Total mercury distribution as a proxy of urban and industrial pollution along the Brazilian coast. Química Nova, v.27, n.5, p. 763-770, 2004.

Mason, R. P., Fitzgerald, W. F. \& Morel, F. M. The biogeochemical cycling of elemental mercury: anthropogenic influences. Geochimica et Cosmochimica Acta, v.58. n.15, p.3191-3198, 1994.

Menezes, M. F. As tartarugas marinhas do Brasil. Arquivos de Ciências do Mar, v.12, n.1, p.17-20, 1972.

Rocha, G. O., Guarieiro, A. L., de Andrade, J. B., Eça, G. F., Aragão, N. M., Aguiar, R. M. \& Hatje, V. Contamination at Todos os Santos Bay [Contaminação na Baía de Todos os Santos], 2012.

Sakai, H., Saeki, K., Ichihashi, H., Suganuma, H., Tanabe, S. \& Tatsukawa, R. Speciesspecific distribution of heavy metals in tissues and organs of loggerhead turtle (Caretta caretta) and green turtle (Chelonia mydas) from Japanese coastal waters. Mar. Pollut. Bull., v. 40, p. 701-709, 2000.

Schneider, L., Maher, W., Green, A. \& Vogt, R.C. Mercury contamination in reptiles: an emerging problem with consequences for wild life and human health. Mercury: Sources Aplications and Health Impacts. Nova Science Publishers, Inc., Hauppauge, New York, USA, p.173-232, 2013.

Toni, M., Dalla Valle, L. \& Alibardi, L. Hard (beta-) keratins in the epidermis of reptiles: Composition, sequence, and molecular organization. J. Proteome Res., v. 6, p.3377-3392, 2007.

UNEP. Global Mercury Assessment 2013: Sources, Emissions, Releases, and Environmental Transport. Unep 42, 2013. 